\title{
Automatic volumetric segmentation of human visual retinotopic cortex
}

\author{
Serge O. Dumoulin, ${ }^{\mathrm{a}, \mathrm{b}, *}$ Richard D. Hoge, ${ }^{\mathrm{c}}$ Curtis L. Baker, Jr., ${ }^{\mathrm{a}}$ Robert F. Hess, ${ }^{\mathrm{a}}$ \\ Rebecca L. Achtman, ${ }^{a}$ and Alan C. Evans ${ }^{\mathrm{b}}$ \\ ${ }^{a}$ McGill Vision Research Unit, Department of Ophthalmology, McGill University, Montréal, Québec, Canada \\ ${ }^{\mathrm{b}}$ McConnell Brain Imaging Centre, Montréal Neurological Institute, McGill University, Montréal, Québec, Canada \\ ${ }^{\mathrm{c}}$ Nuclear Magnetic Resonance Center, Massachusetts General Hospital, Charlestown, MA, USA
}

Received 17 May 2002; revised 11 September 2002; accepted 21 October 2002

\begin{abstract}
Previous identification of early visual cortical areas in humans with phase-encoded retinotopic mapping techniques have relied on an accurate cortical surface reconstruction. Here a 3D phase-encoded retinotopic mapping technique that does not require a reconstruction of the cortical surface is demonstrated. The visual field sign identification is completely automatic and the method directly supplies volumes for a region-of-interest analysis, facilitating the application of cortical mapping to a wider population. A validation of the method is provided by simulations and comparison to cortical surface-based methodology.
\end{abstract}

(C) 2003 Elsevier Science (USA). All rights reserved.

Keywords: fMRI; Retinotopy; Visual areas; Vision; Human cortex; Brain mapping

\section{Introduction}

The cortex contains many separate regions that are involved in different processes and their localization aids functional studies of cortical neuronal mechanisms. For example, the localization of cortical areas allows the possibility of using a volume (or region) of interest (VOI/ROI) analysis, thereby improving signal-to-noise ratio (SNR) due to intra- and intersubject averaging. This offers an advantage over previous stereotaxic based averaging methods, by ensuring that voxels are averaged only within the same functional visual areas.

All early visual cortical areas contain a complete retinotopic map of the visual field (see Fig. 1 for a schematic diagram). These areas can be distinguished on the basis of a number of differences in their retinotopy. For example, the visual field representation can be mirror or nonmirror symmetric (Sereno et al., 1994, 1995), and the borders can occur at either the horizontal or the vertical meridia (DeYoe et al.,

\footnotetext{
* Corresponding author. McGill Vision Research Unit, Department of Ophthalmology, 687 Pine Avenue West, H4-14, Montréal, Québec H3A 1A1, Canada. Fax: +1-514-843-1691.

E-mail address: serge@bic.mni.mcgill.ca (S.O. Dumoulin).
}

1996; Engel et al., 1997; Felleman and Van Essen, 1991; Fox et al., 1987; Hasnain et al., 1998; Holmes, 1945; Horton and Hoyt, 1991; Rosa et al., 1993; Shipp et al., 1995).

A method that has been developed to take advantage of the retinotopic organization of early visual areas is phaseencoded retinotopic mapping. This method was made possible by the finding that time delays (or phase lags) of the cortical activity elicited by slowly expanding circular patterns depend on visual field location (Engel et al., 1994). Sereno et al. (1995) were the first to map visual areas using this phase-encoded method and both expanding rings and rotating wedge stimuli. By combining these maps on a cortical surface, Sereno et al. (1995) were able provide an objective criterion of the visual borders by the identification of visual field signs of areas V1, V2, V3, VP, and V4v using a nomenclature previously established from research in nonhuman primates (Felleman and Van Essen, 1991; Rosa et al., 1993). This procedure did not require a significance threshold, and could be implemented in an automatic way. These results were replicated by DeYoe et al. (1996) and Engel et al. (1997), also utilizing a cortical surface-based analysis but manually distinguishing the borders based on polar-angle information. Further phase-encoded mapping 

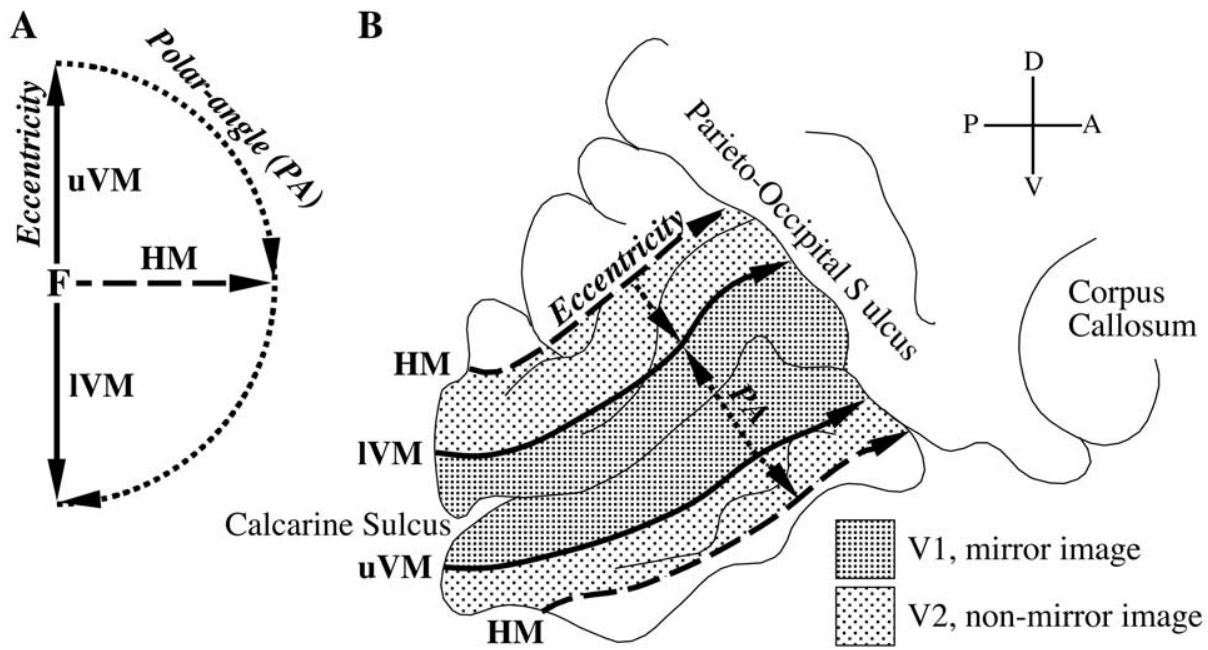

Fig. 1. Schematic diagram of the form of Engel et al. (1997) of the right visual field (A) and cortical representations of areas V1 and V2 on the left hemisphere (B). (A) Right part of the visual field where the fixation (F), upper and lower part of the vertical meridian (uVM, 1VM), and horizontal meridia (HM) are indicated. (B) Schematic drawing of the medial view of the left occipital lobe. The locations of anatomical structures have been indicated for orientation purposes. Drawn are the retinotopic maps of areas V1 and V2 with the corresponding the eccentricity and polar-angle axis and HM and VM representations.

studies with modifications of the stimulus revealed visual areas V3A (DeYoe et al., 1996; Tootell et al., 1997, 1998c), V3B (Press et al., 2001; Smith et al., 1998), V7 (Mendola et al., 1999; Press et al., 2001; Tootell et al., 1998a,c; Tootell and Hadjikhani, 2001), V8 (Hadjikhani et al., 1998), a putative homologue of the lateral intraparietal area (LIP) (Sereno et al., 2001), and retinotopic organization in human MT/V5 (Huk et al., 2002).

Both versions of the above phase-encoded retinotopic mapping method rely upon a reconstructed cortical surface derived from anatomical MRI data. Potentially, this could cause problems not only because there could be errors in the surface reconstruction, but also because of the different spatial resolutions of anatomical and functional images. Furthermore, even if the cortical surface is accurately reconstructed, there is a problems of interpolating a 3D volume onto a 2D surface, especially if a given voxel intersects twice with the surface or not at all.

Here we present a volumetric method that extracts early retinotopically mapped visual areas in a completely automatic way. More importantly, it does not require an explicit reconstruction of the cortical surface, thereby bypassing any potential problems to do with surface reconstruction thus greatly simplifying the analysis. Assuming the primary goal of the visual area identification is to define a VOI, then this new algorithm achieves this without the intermediate cortical surface reconstruction/resampling step.

\section{Methods}

\subsection{Magnetic resonance imaging}

The magnetic resonance images were acquired with a Siemens Magnetom Vision 1.5T MRI. The experiments were conducted with a surface coil (circularly polarized, receive-only) centered over their occipital pole. Head position was fixed by means of a foam headrest and a bite-bar.

T1-weighted anatomical MR images (aMRI) were acquired prior to the functional scans. This aMRI utilized a 3D gradient echo $(\mathrm{GE})$ sequence $(\mathrm{TR}=22 \mathrm{~ms}, \mathrm{TE}=10 \mathrm{~ms}$, flip angle $=30$ degrees) and yielded $80256 \times 256$ saggital slices images $1 \times 1 \times 2$-mm voxels.

Multislice T2*-weighted GE echo-planar imaging (EPI) functional MR images $(\mathrm{TR} / \mathrm{TE}=3 / 51 \mathrm{~ms}$, flip angle $=90$ degrees, No. slices $=25$, slice thickness $=4 \mathrm{~mm}$ ) were acquired with a $64 \times 64$ acquisition matrix and a $256 \times 256$ rectangular field of view, providing a voxel resolution of 4 $\mathrm{mm}^{3}$. The slices were taken either parallel or perpendicular to the calcarine sulcus. For each dynamic scan, 128 measurements (time frames) were acquired, giving a total scanning time of approximately $6.5 \mathrm{~min}$. Six to eight dynamic scans were performed in each session. In a separate session T1-weighted aMRI, images were acquired with a head-coil (circularly polarized, transmit and receive), also with a 3D GE sequence, yielding $170256 \times 256$ sagittal images comprising $1-\mathrm{mm}^{3}$ voxels.

Seven subjects were used ( 1 female; mean age: 34 , age range: 25-48). All observers had normal or corrected to normal visual acuity. All studies were performed with the informed consent of the subjects and were approved by the Montréal Neurological Institute Research Ethics Committee.

\subsection{Visual stimuli}

The visual stimuli were generated on a Silicon Graphics O2 computer with OpenGL-based software and displayed with an LCD projector (NEC Multisync MT820). The stimuli were presented on a rear-projection screen placed in the bore, which was viewed by means of a mirror mounted 


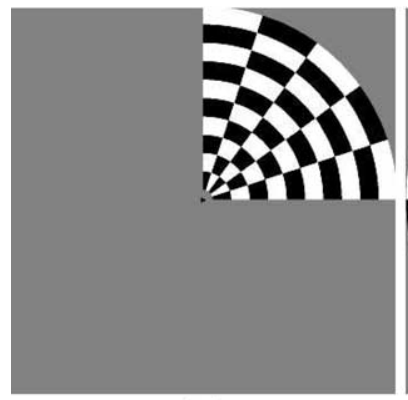

(a)

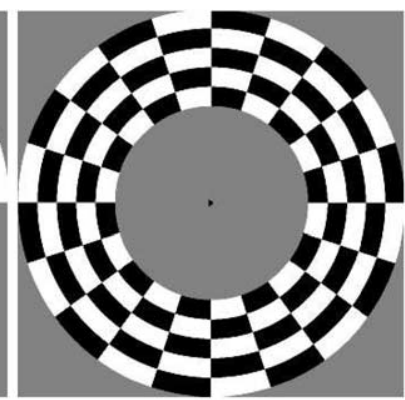

(b)
Fig. 2. Spatial layout of the visual stimuli used. Stimuli used for phaseencoded retinotopic mapping of polar-angle (a) and eccentricity (b). The checks in the wedge and annulus were contrast reversing at $8 \mathrm{~Hz}$. The entire wedge and annulus were rotating and expanding, respectively, at a rate of $0.03 \mathrm{~Hz}$.

above the eyes of the subject. The total visual display subtended 34 degrees.

In the center of each stimulus was a fixation triangle, subtending 0.2 degree, randomly changing during the scan to point either left or right. After each functional timeframe, i.e., every $3 \mathrm{~s}$, the subjects indicated the direction of the triangle by means of a mouse-press. This task ensured fixation of the subjects and controlled their attention.

Standard stimuli were used to create polar-angle and eccentricity maps of the visual cortex (Engel et al., 1994; Sereno et al., 1995; DeYoe et al., 1996; Engel et al., 1997). Rotating wedge and expanding annulus sections of a radial dynamic checkerboard were used for the phase-encoded retinotopic mapping (Figs. 2a and 2 b). Both stimuli completed a full cycle in 12 time frames $(0.03 \mathrm{~Hz})$, giving a total of 10 cycles per scanning run. The checkerboard had a contrast of $100 \%$, which was contrast-reversing at $4 \mathrm{~Hz}$. The wedge subtended 90 degrees.

\subsection{Data analysis}

\subsubsection{Anatomical images}

The global T1-weighted aMRI scans were corrected for intensity nonuniformity (Sled et al., 1998; Arnold et al., 2001) and automatically registered (Collins et al., 1994) in a stereotaxic space (Talairach and Tournoux, 1988) using a stereotaxic model of 305 brains (Evans et al., 1992). The surface-coil aMRI, acquired in the same session as the functional images, was aligned with the head-coil aMRI, thereby allowing an alignment of the functional data with a head-coil MRI and subsequently stereotaxic space. This alignment was performed with an automated script combining correction for the intensity gradient in the surface-coil aMRI (Sled et al., 1998) and intrasubject registration (Collins et al., 1994). A validation of this method was described in a previous study (Dumoulin et al., 2000). The aMRIs were classified into gray matter, white matter, and CSF (Kollokian, 1996; Zijdenbos et al., 1998), after which two cortical surfaces were simultaneously reconstructed at the inner and outer edge of the cortex (MacDonald et al., 2000). The surface-normals of the cortical models were smoothed to produce an "unfolded" model of the cortical sheet (MacDonald et al., 2000). All processing steps were completely automatic and all the data are presented in a stereotaxic space (Talairach and Tournoux, 1988; Collins et al., 1994).

\subsubsection{Preprocessing of functional images}

The first eight time frames of each functional run were discarded due to start-up magnetization transients in the data. All remaining scans in each functional run were normalized for spatial slice-intensity variations by multiplying each slice by a constant factor across all time frames. The functional data were blurred with an isotropic 3D Gaussian kernel (full-width half-maximum (fwhm) $=6 \mathrm{~mm}$ ) to attenuate high-frequency noise, and to get a more robust minimization of the motion correction algorithm (Woods et al., 1992, 1998). Two of the subjects' (SD and CB) functional runs contained high-amplitude spurious spikes; these spikes interfere with the VFS computation and were removed by a median filter (width three time points) in the time domain. The functional scans were corrected for subject motion within and between scans using the AIR package (Woods et al., 1992, 1998; Jiang et al., 1995).

\subsubsection{Volumetric visual field sign identification}

A flow chart illustrating the method of volumetric visual field sign identification is shown in Fig. 3. The data were analyzed in a stereotaxic space (Collins et al., 1994; Talairach and Tournoux, 1988), at a high resolution $\left(1 \mathrm{~mm}^{3}\right)$ using an average of all preprocessed functional runs. A slice through the left hemisphere $(y=-10)$ in stereotaxic space is shown for the volumes created at different stages. At the top of the flow chart, the input images are shown: the T1-weighted anatomical MRI and two average preprocessed fMRIs of responses to each of the two stimuli used (see Fig. 2).

The power spectrum of each voxel's time series was computed using a discrete Fourier transform and used in the construction of four preliminary maps, i.e., two phase maps, a magnitude map, and a $t$-statistical map (see Fig. 3, row 2). The phase maps were created by taking the phase of the fundamental frequency (i.e., 10 cycles/scan) of the fMRI response. The phases of the fundamental frequency varied as a function of polar angle when the stimulus was the rotating wedge and as a function of eccentricity when subjects viewed expanding annuli. For the creation of the magnitude map and the $t$-statistical map data of the two fMRI scans were combined. Magnitude maps were generated by dividing the amplitude of the fundamental component of the fMRI response by the average amplitude of frequencies assumed to contain noise (typically 40-60 cycles/scan). $t$-statistical maps were created using a Spearman rank order test for each voxel, where the phase of the design matrix is taken from the corresponding phase map. Both the magnitude map and the $t$-statistical map provide types of signalto-noise maps which are used to weight the data. 


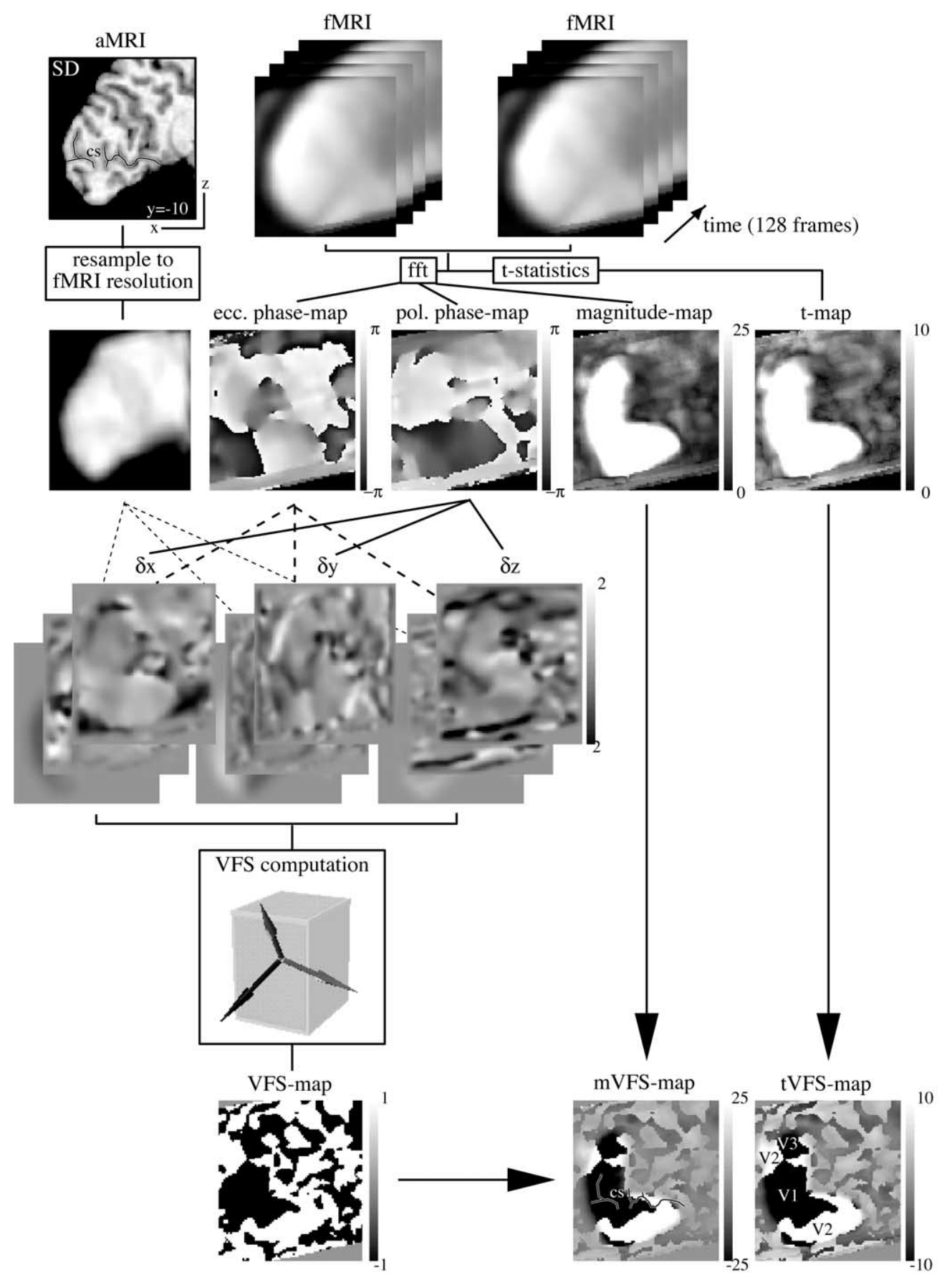

Fig. 3. A flow chart describing the method with examples of slices through volumes created by the different processing steps. On the top of the flow chart the three input MR images are shown (aMRI and two fMRI scans); bottom right shows two output images (mVFS and tVFS). On the input anatomical scan and the mVFS map the calcarine sulcus (CS) is indicated with black and white lines for orientation purposes. On the tVFS map the different visual areas are labeled.

To define for each voxel a 3D-vector orthogonal to the rate of change in the phase maps, the $\delta x, \delta y$, and $\delta z$ partial directional derivatives were computed. The $\delta x, \delta y$, and $\delta z$ partial derivatives of the phase maps were created by convolving the volumes with the partial derivative of a Gaussian kernel (typically, fwhm $=3 \mathrm{~mm}$ ). Due to the circular nature of phase, the phase maps contain $\pi$ to $-\pi$ reversals, and the derivative vector for these voxels will point orthog- onal to the $\pi /-\pi$ change which is opposite to the actual phase change. To avoid these $\pi /-\pi$ artifacts other sets of phase maps are created from the original set by shifting the phase (typically by $0.5 \pi$ ). Thus in the resulting phase maps (typically 4) the $\pi /-\pi$ shift occurs at a different location. The $\pi /-\pi$ artifacts can be identified on a voxel by voxel basis due to their opposite polarities when comparing the derivatives of the phase maps. Selective averaging (or by 


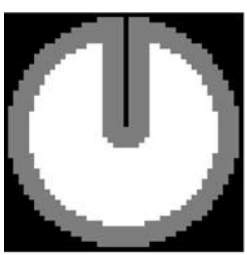

(a)

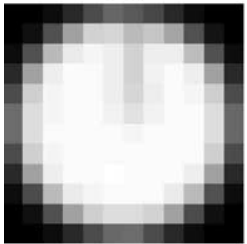

(e)

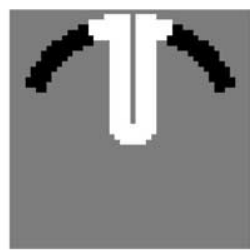

(b)

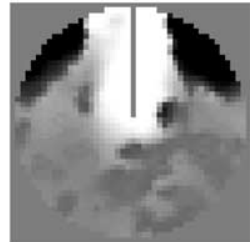

(f)

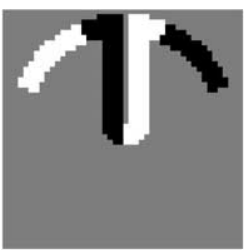

(c)

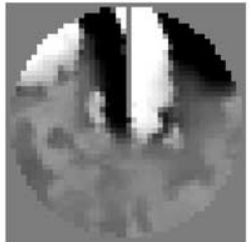

(g)

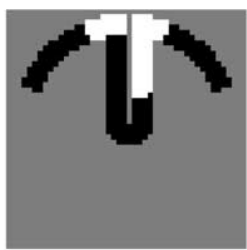

(d)

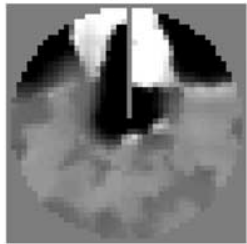

(h)

Fig. 4. Slices from volumes created during simulations. (a) Anatomical volume. (b-d) Three simulated visual field sign maps used to generate the functional data. (e) Lower resolution preprocessed functional time frame. (f-h) Corresponding reconstructed mVFS maps.

taking the median) of the partial derivative sets for each voxel removes the $\pi /-\pi$ artifacts, resulting in one partial derivative set for polar angle and eccentricity (see Fig. 3, row 3).

To prevent aliasing due to the resolution differences of the aMRI and the fMRI datasets, the anatomical MRI was resampled to the resolution of the functional images (see Fig. 3, column 1). Partial derivative volumes were also generated from the anatomical MRI; these derivative vectors identify the cortical surface normals (see Fig. 3, row 3).

The relative directions of these three orthogonal derivative vectors (polar angle, eccentricity, and cortex) identify the visual field sign (VFS) of each voxel (Fig. 3, row 4; an example of the three vectors within a voxel) resulting in a VFS map (Fig. 3, row 5; -1 for mirror image, 1 for non-mirror image, and 0 if the the field sign could not be determined). More specifically, for each voxel the derivative vectors for eccentricity and polar angle were projected onto the plane defined by the anatomic normal vector, which should be tangential to the cortex. Next, the vector cross product of the tangential gradient eccentricity and polar angle vectors is computed, and the sign of the cross product is the visual field sign (Sereno et al., 1994).

To create a weighted map of the VFS computation, the VFS map is multiplied by either the magnitude map or the $t$-statistical map. The absolute values in the resulting maps (mVFS or tVFS, respectively) indicate the SNR or statistical certainty of the VFS computation (see Fig. 3, last row) and both SNR maps are very similar. Before this multiplication all values below 0 are set to 0 in the $t$-statistical map to prevent VFS reversals.

\subsection{Simulations}

To validate the data analysis, simulated data sets were used to test the method in a controlled environment and assess the dependence on different variable values. To evaluate the results of the analysis objectively, besides a visual inspection, a correlation coefficient $\left(r_{\mathrm{xy}}\right)$ of the predefined and reconstructed VFS maps was computed:

$$
r_{x y}=\frac{\sum_{i=1}^{n}\left(x_{i} y_{i}\right)}{\sqrt{\sum_{i=1}^{n} x_{i}^{2} \sum_{i=1}^{n} y_{i}^{2}}}
$$

where $x$ is the predefined VFS map from which the data were simulated and $y$ is the mVFS map reconstructed by the method. The magnitude of the signal in the reconstructed maps may vary due to resampling to a lower resolution and spatial smoothing. Only voxels with a predefined field sign and their corresponding voxels in the mVFS maps were included in the $r_{x y}$ computation. The relevant values of $r_{x y}$ range between 0 (no correlation between the two maps) and 1 (exact reconstruction of the predefined map).

A simple spherical model was constructed at a $1-\mathrm{mm}^{3}$ resolution with a single sulcus (see Fig. 4a). The dimensions of the model were chosen to be roughly similar to the occipital lobe with the calcarine sulcus in a stereotaxic space (Collins et al., 1994; Talairach and Tournoux, 1988). In this model retinotopically mapped regions were defined with different field signs. Simulated fMRI data were generated from the predefined map, at a spatial $\left(4 \mathrm{~mm}^{3}\right)$ and temporal (3s) resolution of the actual fMRI scans (see Fig. $4 \mathrm{e}$, after blurring). Each functional time series had a mean value, determined by the anatomical model; added to this voxel were Gaussian noise and, if present, an fMRI signal. The fMRI signal of a particular phase was computed by convolving the block design with a hemodynamic response model (Boynton et al., 1996). The variance of the zero-mean Gaussian noise was $2 / 3$ the maximum simulated fMRI sig- 


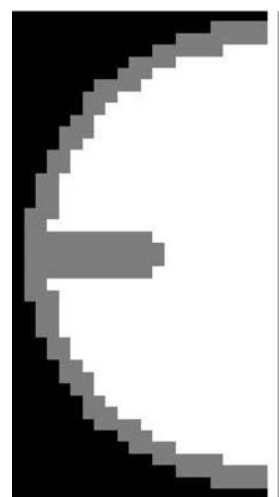

(a)

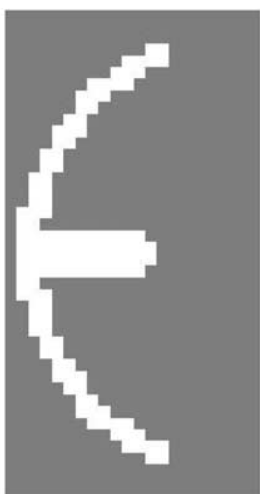

(b)

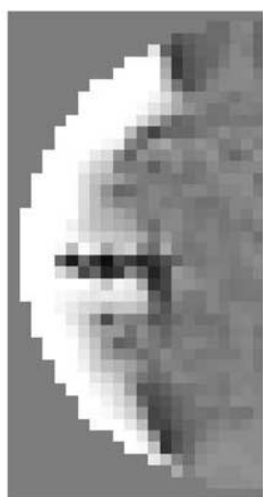

(c)

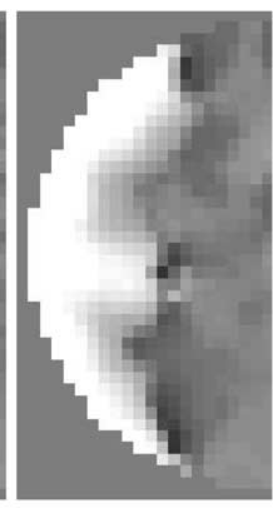

(d)

Fig. 5. Simulated volumes. (a, b) Slice through the anatomical volume and predefined VFS map, respectively. (c, d) Reconstructed VFS map without and with matching the spatial resolution of the functional and anatomical data.

nal amplitude. The resulting signal-to-noise ratio in the simulated data as indicated by the magnitude maps was about $30 \%$ worse than the real data. Thus the simulated data represented a worst-case scenario. The simulated data sets were analyzed the same way as the real fMRI data.

\section{Results}

\subsection{Simulations}

Results of the simulation are shown in Fig. 4. The slices are taken through the middle of the spherical model, orthogonal to the sulcus. Fig. 4a shows the anatomical model. The functional data were simulated according to the visual field layouts of Figs. $4 \mathrm{~b}-4 \mathrm{~d}$ (VFSs are either 1 or -1 ). The volume in Fig. $4 \mathrm{~b}$ simulates the textbook layout of cortical areas V1 and V2, while Figs. 4c and 4d represent more complex versions of cortical area layouts. A slice through one time frame of a preprocessed (blurred) functional data set of lower spatial resolution is shown in Fig. 4e. Figs $4 \mathrm{~g}-4 \mathrm{~h}$ show the resulting visual field sign map multiplied by the computed magnitude map (image values range between -7.5 and 7.5).

Note that all areas and corresponding visual field signs were accurately reconstructed. The average correlation coefficient and standard deviation (see Eq. (1)) for reconstructed mVFS maps as shown in Figs. $4 \mathrm{~g}-4 \mathrm{~h}$ was $0.89 \pm$ $0.03,0.82 \pm 0.03$, and $0.70 \pm 0.00$, respectively $(n=4)$. The decreasing values are due to an increasing amount of borders between predefined areas. Even though the relative topography of all areas are accurately reconstructed, the exact border locations may vary due to partial volume effects and spatial smoothing of the data, degrading the correlation coefficient.

The different spatial resolution for the anatomical and functional datasets could interfere with the identification of the visual field signs. Take, for instance, a functional voxel with a specific polar and eccentricity angle located in a narrow or small sulcus, which is accurately represented in an aMRI. When analyzed at the spatial resolution of the aMRI data, parts of this voxel will fall on opposite banks of the sulci and one location will be a mirror image of the other. Therefore they will be assigned different field signs and may be interpreted as different visual areas. This problem is illustrated in Fig. 5. Figs. 5a and 5b show the anatomical volume with a small sulcus and the predefined VFS map, respectively. Fig. 5c displays the resulting mVFS map showing the incorrectly labeled lower side of the sulcus. In Fig. $5 \mathrm{~d}$ this problem is solved by matching the functional and anatomical spatial resolutions. This is a problem that could also occur with methods that depend on a cortical surface reconstruction even if the cortical surface is accurately reconstructed.

\subsection{Volumetric visual field sign identification}

Examples of slices through resulting VFS maps are shown in Figs. 6 and 7. In both cases the VFS maps are weighted by the corresponding $t$-statistical maps; i.e., the absolute value indicates the statistical accuracy of the VFS calculation (tVFS). In both figures, neighboring areas with different field signs can be distinguished, and are identified based on (1) their field sign, (2) what part of the visual field is represented, (3) their relative organization, and (4) their anatomical locations; e.g., V1 is known to be at least partially located within the calcarine sulcus (Gilissen et al., 1995; Gilissen and Zilles, 1996; Rademacher et al., 1993; Stensaas et al., 1974). Thus the tVFS segmentation of the visual cortex is completely automatic, but not the identification of the visual areas, which has to be done manually. The locations of the calcarine sulcus (CS) and parietooccipital sulcus (POS) are indicated in the saggital slices of Fig. 6.

Inspection of Fig. 6 provides a validation of the method described here. In the calcarine sulcus a large region of 

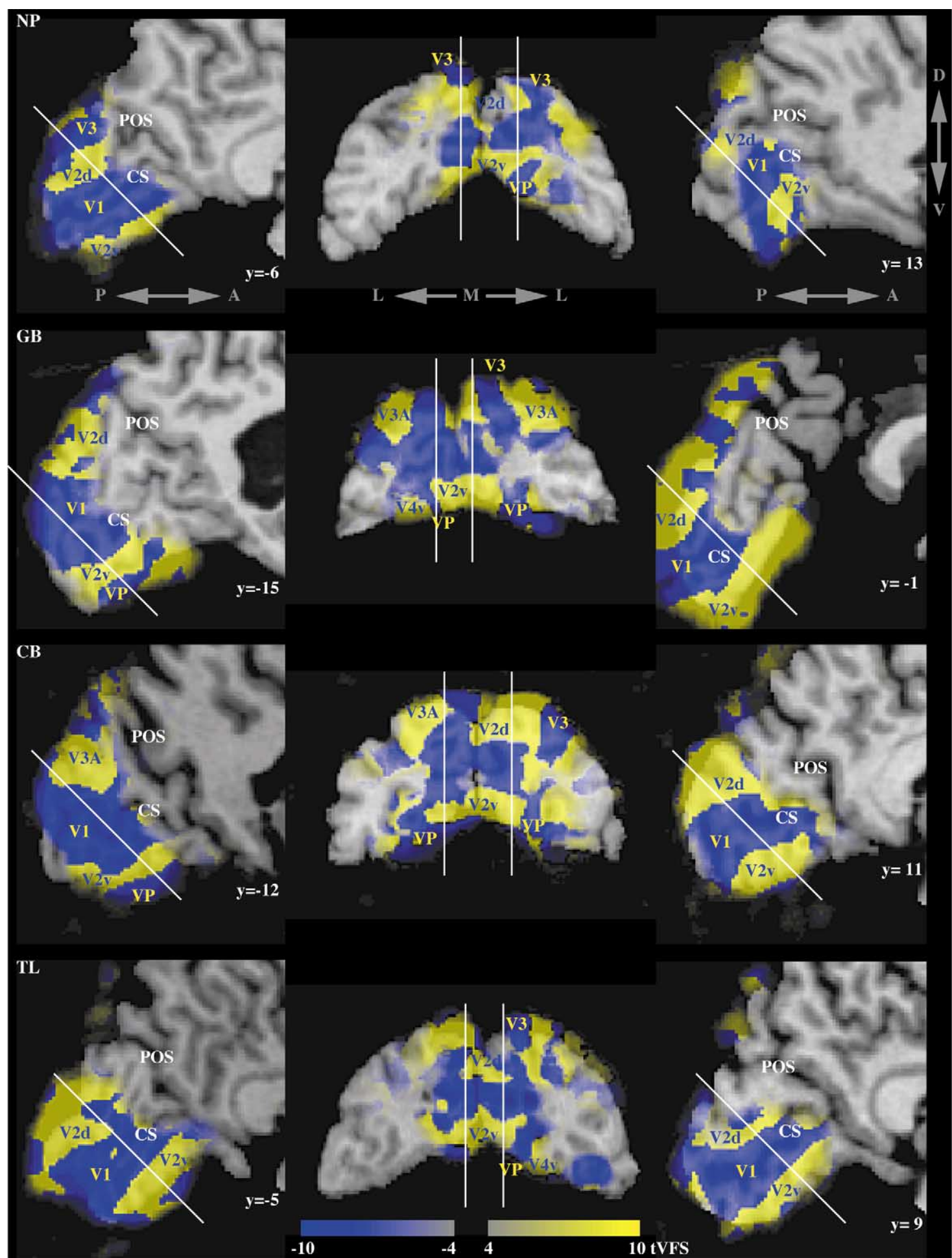

Fig. 6. Visual Field Sign (VFS) maps are shown overlaid on the corresponding anatomical images for four subjects in a stereotaxic space, weighted by the corresponding $t$-statistical maps (tVFS). For each subject two sagittal slices through each hemisphere and an oblique slice are shown. The location of the slices is indicated with the white lines; for the sagittal slices the Talairach coordinates are given. Blue and yellow correspond to opposite field signs, mirror and nonmirror image, respectively. Different visual areas can be identified and are labeled in the volumes. CS, POS, location of the calcarine sulcus and parieto-occipital sulcus, respectively.

negative field sign (mirror image) is present, around which regions opposite field sign can be distinguished corresponding to the known layout of areas V1 and V2 (Amunts et al.,
2000; Clarke and Miklossy, 1990; Gilissen et al., 1995; Gilissen and Zilles, 1996; Holmes, 1945; Horton and Hoyt, 1991; Rademacher et al., 1993; Stensaas et al., 1974; Wong 

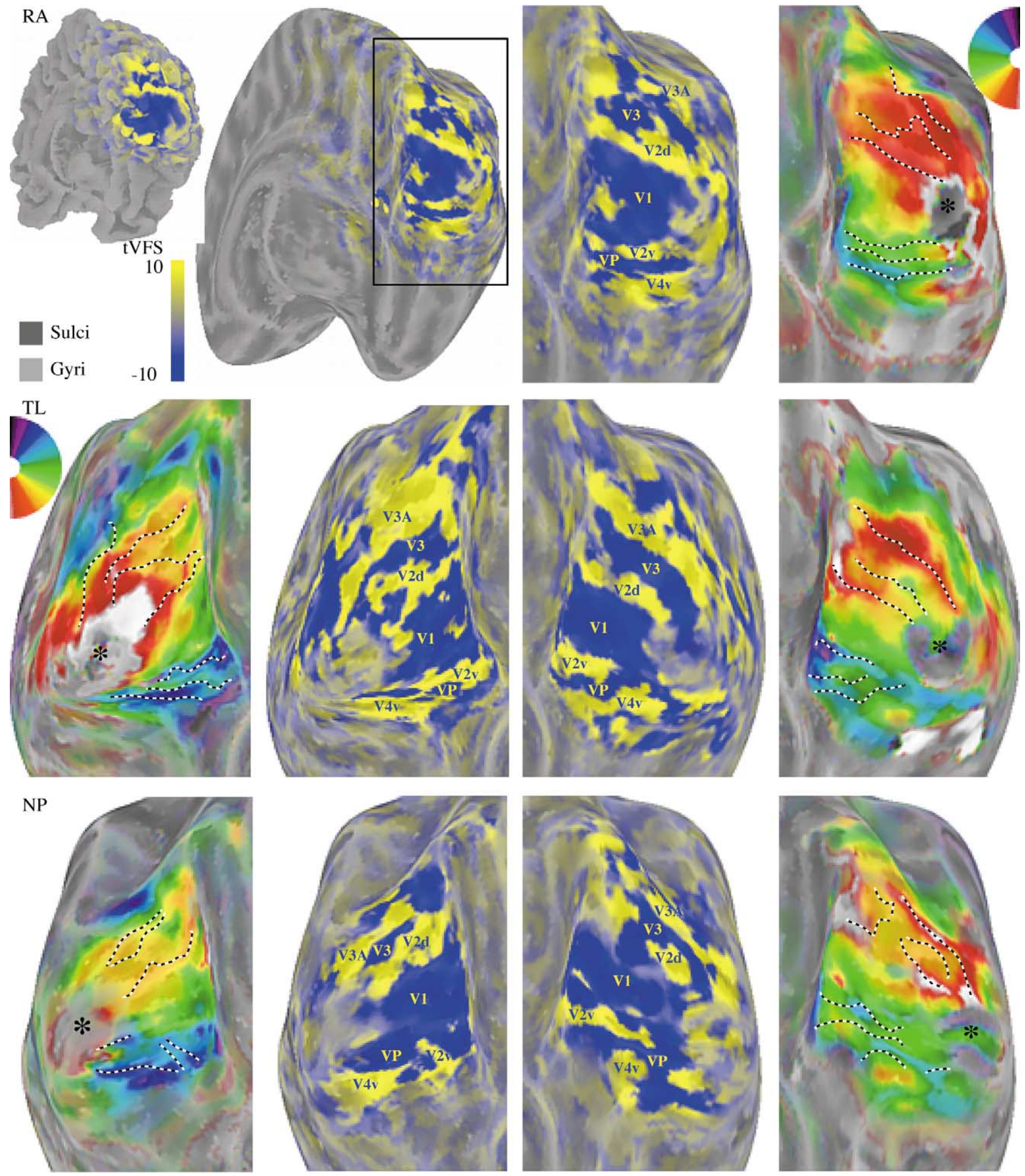

Fig. 7. tVFS images are shown overlaid on the corresponding unfolded cortical surfaces of three subjects. All surfaces are viewed from a medio-posterior viewpoint. For the first subject (RA) the whole folded and unfolded cortical surfaces are shown to facilitate orientation on the other enlarged views. Sulci are colored darker gray then the gyri. For subject RA only the right hemisphere is shown, for the other subjects both hemispheres are presented. Besides surfaces overlaid with the tVFS maps, the same surfaces are shown overlaid with colored polar-angle phase maps are in identical views. The intensity of the phase maps is weighted by the $t$-statistical maps identical to the tVFS maps. The black and white dashed lines indicate borders between visual areas derived from the tVFS maps. The asterisk indicates the cortical representation of the fovea.

and Sharpe, 1999). In a similar fashion other areas can be distinguished.

For comparison with conventional methods, the tVFS maps are shown on reconstructed unfolded cortical surfaces (MacDonald et al., 2000) in the middle columns of Fig. 7. The cortical surface was extracted halfway between the 
Table 1

Correlation coefficients comparing mVES maps of the same subject for three conditions using two masks

\begin{tabular}{llll}
\hline Comparison & fwhm & Avg. occ. lobe mask & Indiv. V1 to V4v mask \\
\hline Average vs single runs $(n=7)$ & 0 & $0.70 \pm 0.09$ & $0.79 \pm 0.06$ \\
& 2 & $0.78 \pm 0.05$ & $0.85 \pm 0.04$ \\
& 4 & $0.83 \pm 0.04$ & $0.89 \pm 0.03$ \\
Single runs, identical slice pos. $(n=3)$ & 6 & $0.86 \pm 0.03$ & $0.91 \pm 0.03$ \\
& 0 & $0.61 \pm 0.06$ & $0.76 \pm 0.04$ \\
& 2 & $0.71 \pm 0.06$ & $0.85 \pm 0.04$ \\
Single runs, different slice pos. $(n=6)$ & 6 & $0.81 \pm 0.04$ & $0.91 \pm 0.03$ \\
& 0 & $0.35 \pm 0.03$ & $0.48 \pm 0.03$ \\
& 2 & $0.45 \pm 0.04$ & $0.58 \pm 0.04$ \\
& 6 & $0.53 \pm 0.04$ & $0.66 \pm 0.04$
\end{tabular}

Note. The results suggest that different slice positions is the primary origin of intrasubject variability. The different levels of spatial smoothing (fwhm) suggest a high reproducibility of the global topography of visual areas.

gray-matter CSF border and the white-matter-gray-matter border. Besides the tVFS maps the corresponding polarangle phase maps are also shown on the unfolded cortical surfaces (left and right columns). The colors of the phase maps correspond to the locations in the visual field as shown in the insets; the intensity of the colors in the phase-maps are also weighted by the $t$-statistical maps in a scale identical to that of the tVFS maps. The black-white dashed lines are the borders of visual areas as derived from the tVFS maps.

Results in Fig. 7 are comparable to those from surfacebased methods (Sereno et al., 1995; DeYoe et al., 1996; Engel et al., 1997). The alternation of visual field signs is in accordance with the known layout of the visual areas. Besides tVFS maps, the polar-angle phase maps are shown in identical views. The representations of the polar-angle maps provide results similar to those of DeYoe et al. (1996) and Engel et al. (1997), where on these kinds of representations the borders were identified manually. The borders on the phase maps in Fig. 7 are derived from the corresponding tVFS maps and fall at the horizontal and vertical meridia, as they are known to occur. For a more quantitative comparison, the VFS were computed on the flattened surfaces (see Fig. 8). The correlation coefficients and standard deviations (see Eq. (1)) between surface and volumetric computed tVFS-maps on the surfaces were $0.50 \pm 0.07(n=6$, for the subjects in Fig. 7) and $0.64 \pm 0.07$ after smoothing (fwhm $=4 \mathrm{~mm}$ ) of the surface-based tVFS map (Chung et al., 2001). Even though the relative topography of all areas are accurately reconstructed, the surface-based tVFS-maps differ due to a dependence on the cortical surface reconstruction, resampling, and processing, and due to resolution differences between the cortical surface and functional data (see Fig. 5).

The visual field sign pattern is in accordance with previous studies identifying visual areas (Sereno et al., 1995; DeYoe et al., 1996; Engel et al., 1997; Tootell et al., 1997; Smith et al., 1998). In all subjects visual areas V1, V2, $\mathrm{V} 3 / \mathrm{VP}, \mathrm{V} 4 \mathrm{v}$, and $\mathrm{V} 3 \mathrm{~A}$ could be identified. The part of the visual field represented in these areas matched with previous studies (see Fig. 7). Parts of visual areas V3B, V7, and V8 could be identified only in some of the subjects (14/14, $13 / 14$, and $9 / 14$ hemispheres, respectively). In the V3B only a representation of one (lower) quadrant of the visual field could be identified; for V7 both an upper and a lower field representation were found. In our results, $\mathrm{V} 4 \mathrm{v}$ contains only a (upper visual field) quadrant representation, and V8 contains a hemifield representation, in agreement with Hadjikhani et al. (1998). For a debate about a different naming scheme and the relationship to macaque V4 see Zeki et al. (1998) and Tootell and Hadjikhani (1998).

A large variability in the location of areas and the borders between them was observed for the different subjects, and even between hemispheres of a given subject. In imaging data in stereotaxic space (Collins et al., 1994; Talairach and Tournoux, 1988) variability may be due to gross anatomical variations, differences in the topographic relationship of gross anatomy and functional areas, and methodological issues (Hunton et al., 1996; Rademacher et al., 1993; Steinmetz et al., 1989, 1990; Steinmetz and Seitz, 1991). To quantify this variability a correlation coefficient, $r_{x y}$ (see Eq. (1)), was computed for voxels of the mVFS maps falling within an average anatomical occipital lobe mask in stereotaxic space (Collins et al., 1994; Talairach and Tournoux, 1988). The mVFS maps were used (1) to weight the data according to signal strength, because the occipital lobe mask will contain more than the identified visual areas, and (2) for consistency with the further interpretations where either the tVFS or the mVFS maps are used. The average correlation coefficient $\left(r_{x y}\right)$ and standard deviation between pairs of individual subjects $(n=7)$ is $0.11 \pm 0.06(n=21)$.

To estimate how much of this variation is due to the method, $r_{x y}$ was also computed over different runs of the same subject (subjects TL and RA, Table 1). Taking one run reduces the amount of signal, and the average $r_{x y}$ and standard deviation comparing the grand average of that subject with each individual run is $0.70 \pm 0.09(n=7$, average occipital lobe mask). The next step compares single fMRI 
runs of the same subject, using data acquired in identical and different slice positions. There were different slice positions because (1) the slices were moved within a scanning session or (2) the runs were acquired in a different scanning sessions altogether, as would occur with different subjects. Computing $r_{x y}$ by comparing single runs, the $r_{x y}$ values were $0.61 \pm 0.06(n=3)$ and $0.35 \pm 0.03(n=6)$ for the same and different slice positions, respectively. These values suggest that the position of the sampling grid (slice positions) is the primary origin of variability within a subject. In each of the individual runs all areas were accurately reconstructed, suggesting that the lowered $r_{x y}$ values result from variations in the exact border locations and size of the areas, as is also supported by the simulation results. This is verified quantitatively by blurring (fwhm 2, 4, and $6 \mathrm{~mm}$ ) the mVFS maps, attenuating the border contribution to the $r_{x y}$ computation, thus resulting in stronger correlations (Table 1). Please note that the $r_{x y}$ values depend on the VOI/ROI over which the $r_{x y}$ was computed. Here the VOI was an average anatomical map of the occipital cortex, so one VOI could be used for different subjects. If only identified areas V1 to V3A and $\mathrm{V} 4 \mathrm{v}$ were used for the within-subjects comparison, an increase of the $r_{x y}$ values was observed (see Table 1).

This within-subject variability is less (larger $r_{x y}$ values) than the variability between subjects $\left(r_{x y}=0.11 \pm 0.06\right)$, notwithstanding that each subject's maps are of a higher quality (SNR) in the between-subjects comparison. Given these two arguments, the data suggest that the methodological variations, as indicated by the within subject comparison, are relatively small and cannot explain the betweensubject variation. Therefore we conclude that the betweensubject variation is primarily due to variations in gross anatomy and variations in the relation between gross anatomy and functional areas.

In the previous analysis T1-weighted aMRI were used, but white matter only images can be used as well, making the analysis independent of the anatomical scan parameters. In the latter case the white matter was automatically identified using the classifier INSECT (Kollokian, 1996; Zijdenbos et al., 1998). Results for both input anatomical images were very similar $\left(r_{x y}=0.86\right.$ for subject RA).

\section{Discussion}

Volumetric retinotopic mapping can be used to identify retinotopically mapped visual areas. This method is automatic, does not require a cortical surface reconstruction, and directly supplies volumes for a region of interest analysis.

All early retinotopic areas up to and including V3A and $\mathrm{V} 4 \mathrm{v}$ could be identified reliably in all subjects $(n=7)$. The layout of the cortical areas is in agreement with previous fMRI (DeYoe et al., 1996; Engel et al., 1997; Sereno et al., 1995; Smith et al., 1998; Tootell et al., 1997), positron emission tomography (Fox et al., 1987; Hasnain et al., 1998,
2001), lesion studies (Holmes, 1945; Horton and Hoyt, 1991; Wong and Sharpe, 1999), and cytoarchitectonic maps of Brodmann's area 17 and 18 (Amunts et al., 2000; Clarke and Miklossy, 1990).

In most subjects, parts of $\mathrm{V} 3 \mathrm{~B}, \mathrm{~V} 7$, and $\mathrm{V} 8$ could also be identified. These areas are at the limit of the current methodology and therefore may be only partially identified, if at all, as evidenced by inconsistent descriptions of several studies (Press et al., 2001; Smith et al., 1998; Tootell et al., 1998a,b). Press et al. (2001) found an upper and lower visual field representation for both V3B and V7. Smith et al. (1998) could determine only a lower field representation for area V3B, while Tootell et al. (1998a,b) could only measure a lower field representation for area V7. Here we can confirm both an upper and a lower field representation for V7; however, for $\mathrm{V} 3 \mathrm{~B}$ only a representation of the lower visual field was found.

A high variability in the location of the visual areas was found between subjects in a stereotaxic space. This is not surprising since various studies have described variations in functional and anatomical patterns in striate and extrastriate cortex (Aine et al., 1996; Amunts et al., 2000; Dumoulin et al., 2000; Gilissen et al., 1995; Gilissen and Zilles, 1996; Hasnain et al., 1998; Rademacher et al.,1993; Roland et al.,1997; Steinmetz et al., 1990; Steinmetz and Seitz, 1991; Stensaas et al., 1974; Watson et al., 1993) The variability found here can mainly be attributed to variations in gross anatomy and variations in the relationship between gross anatomy and functional areas, rather than methodological differences.

Surface-based analysis, display, and 2-D coordinate systems have been proposed (Drury et al., 1996; Fischl et al., 1999a,b; Van Essen et al., 1998, 2000; Van Essen and Drury, 1997). Indeed cortical surfaces are now widely used for display purposes, including this study. Our method does not argue against surface-based methods. It provides a methodological simplification for retinotopic mapping applications, especially for volume-of-interest analysis, by avoiding potential problems with cortical surface reconstruction and resampling. The data can still be presented on a cortical surface but the analysis is not limited by it; i.e., the analysis does not depend on its accurate, resolutionmatched, reconstruction.

The method described here provides an automatic volumetric segmentation of early visual areas, which is comparable to conventional surface-based methods. This offers the advantage of not requiring any manual interference and directly supplying VOIs thereby facilitating the application of cortical mapping to a wider population. Furthermore, due to its completely automatic analysis and decreased processing time by bypassing cortical surface reconstruction, this method offers the possibility of near real-time retinotopic mapping (for example, see near real-time creations of phase maps in Voyvodic, 1999). 


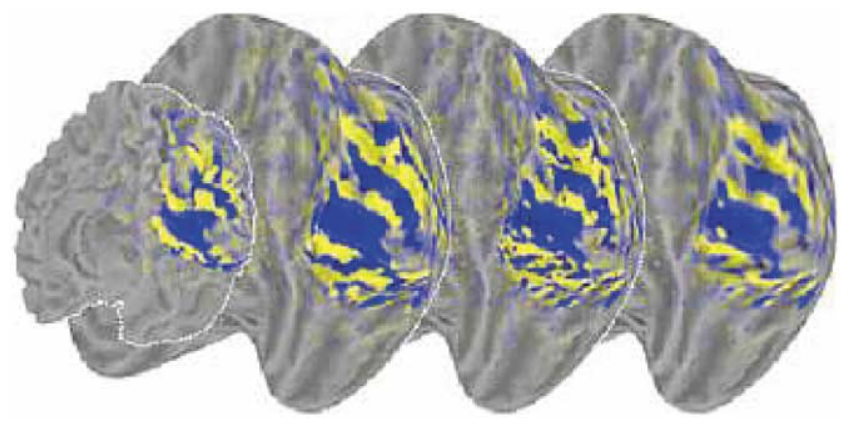

Fig. 8. A comparison of volumetric VFS computation with a surface-based VFS computation. Three unfolded tVFS maps are displayed on the corresponding cortical surface of one subject (TL) in a view similar to that in Fig. 7. The whole folded cortical surface is shown to facilitate orientation on the other unfolded surfaces. The left unfolded surface shows the tVFS map resulting from the volumetric VFS computation. The right tVFS maps are resulting from a surface-based VFS computation, where the far right one has been smoothed ( $\mathrm{fwhm}=4 \mathrm{~mm}$ )

\section{Acknowledgments}

The authors thank Gareth Barnes, Bruce Pike, and Louis Collins for help in various stages of the project. This research was supported by Natural Sciences and Engineering Research Council of Canada (NSERC) Grant OGP0001978 to C.L.B., Canadian Institutes of Health Research (CIHR) Grant MT 10818 and NSERC Grant 0GP0046528 to R.F.H., and International Consortum for Brain Mapping (ICBM) Grant MH52176-06 to A.C.E. The software package is freely available at http://www.bic.mni.mcgill.ca/users/ serge/.

\section{References}

Aine, C.J., Supek, S., George, J.S., Ranken, D., Lewine, J., Sanders, J., Best, E., Tiee, W., Flynn, E.R., Wood, C.C., 1996. Retinotopic organization of human visual cortex: departures from the classical model. Cerebral Cortex 6, 354-361.

Amunts, K., Malikovic, A., Mohlberg, H., Schormann, T., Zilles, K., 2000. Brodmann's areas 17 and 18 brought into stereotaxic space-where and how variable? NeuroImage 11, 66-84.

Arnold, J.B., Liow, J.-S., Schaper, K.A., Stern, J.J., Sled, J.G., Shattuck, D.W., Worth, A.J., Cohen, M.S., Leahy, R.M., Mazziotta, J.C., Rottenberg, D.A., 2001. Qualitative and quantitative evaluation of six algorithms for correcting intensity nonuniformity effects. NeuroImage 13, 931-943.

Boynton, G.M., Engel, S.A., Glover, G.H., Heeger, D.J., 1996. Linear systems analysis of functional magnetic resonance imaging in human V1. J. Neurosci. 16, 4207-4221.

Chung, M.K., Worsley, K.J., Taylor, J., Ramsay, J., Robbins, S., Evans, A.C., 2001. Diffusion smoothing on the cortical surface. NeuroImage 13, S95.

Clarke, S., Miklossy, J., 1990. Occipital cortex in man: organization of callosal connections, related myelo- and cytoarchitecture, and putative boundaries of functional visual areas. J. Comp. Neurol. 298, 188-214.

Collins, D.L., Neelin, P., Peters, T.M., Evans, A.C., 1994. Automatic 3D intersubject registration of MR volumetric data in standardized Talairach space. J. Comput. Assist. Tomogr. 18, 192-205.
DeYoe, E.A., Carman, G.J., Bandettini, P., Glickman, S., Wieser, J., Cox, R., Miller, D., Neitz, J., 1996. Mapping striate and extrastriate visual areas in human cerebral cortex. Proc. Natl. Acad. Sci. USA 93, 23822386.

Drury, H.A., Van Essen, D.C., Anderson, C.H., Lee, C.W., Coogan, T.A., Lewis, J.W., 1996. Computerized mappings of the cerebral cortex: a multiresolution flattening method and a surface-based coordinate system. J. Cogn. Neurosci. 8, 1-28.

Dumoulin, S.O., Bittar, R.G., Kabani, N.J., Baker Jr, C.L., Le Goualher, G., Pike, G.B., Evans, A.C., 2000. A new anatomical landmark for reliable identification of human area V5/MT: a quantitative analysis of sulcal patterning. Cerebral Cortex 10, 454-463.

Engel, S.A., Glover, G.H., Wandell, B.A., 1997. Retinotopic organization in human visual cortex and the spatial precision of functional MRI. Cerebral Cortex 7, 181-192.

Engel, S.A., Rumelhart, D.E., Wandell, B.A., Lee, A.T., Glover, G.H., Chichilnisky, E.J., Shadlen, M.N., 1994. FMRI of human visual cortex. Nature 369, 525.

Evans, A.C., Collins, D.L., Milner, B., 1992. An MRI-based stereotaxic atlas from 250 young norma subjects. Soc. Neurosci. Abstr. 18, 408.

Felleman, D.J., Van Essen, D.C., 1991. Distributed hierarchical processing in the primate cerebral cortex. Cerebral Cortex 1, 1-47.

Fischl, B., Sereno, M.I., Dale, A.M., 1999a. Cortical surface-based analysis: II. Inflation, flattening, and a surface-based coordinate system. NeuroImage 9, 195-207.

Fischl, B., Sereno, M.I., Tootell, R.B., Dale, A.M., 1999b. High-resolution intersubject averaging and a coordinate system for the cortical surface. Human Brain Mapping 8, 272-284.

Fox, P.T., Miezin, F.M., Allman, J.M., Van Essen, D.C., Raichle, M.E., 1987. Retinotopic organization of human visual cortex mapped with positron-emission tomography. J. Neurosci. 7, 913-922.

Gilissen, E., Iba-Zizen, M.-T., Stievenart, J.-L., Lopez, A., Trad, M., Cabanis, E.A., Zilles, K., 1995. Is the length of the calcarine sulcus associated with the size of the human visual cortex? A morphometric study with magnetic resonance tomography. J. Brain Res. 36, 451-459.

Gilissen, E., Zilles, K., 1996. The calcarine sulcus as an estimate of the total volume of the human striate cortex: A morphometric study of reliability and intersubject variability. J. Brain Res. 37, 57-66.

Hadjikhani, N., Liu, A.K., Dale, A.M., Cavanagh, P., Tootell, R.B.H., 1998. Retinotopy and color sensitivity in human visual cortical area V8. Nature Neurosci. 1, 235-241.

Hasnain, M.K., Fox, P.T., Woldorff, M.G., 1998. Intersubject variability of functional areas in the human visual cortex. Human Brain Mapping 6, 301-315.

Hasnain, M.K., Fox, P.T., Woldorff, M.G., 2001. Structure-function spatial covariance in the human visual cortex. Cerebral Cortex 11, 702716.

Holmes, G., 1945. The organization of the visual cortex in man. Proc. R. Soc. London 132, 348-361.

Horton, J.C., Hoyt, W.F., 1991. Quadrantic visual field defects: A hallmark of lesions in extrastriate (V2/V3) cortex. Brain 1114, 1703-1718.

Huk, A.C., Dougherty, R.F., Heeger, D.J., 2002. Retinotopy and functional subdivision of human areas MT and MST. J. Neurosci. 22, 7195-7205.

Hunton, D.L., Miezin, F.M., Buckner, R.L., van Mier, H.I., Raichle, M.E., Petersen, S.E., 1996. An assessment of functional-anatomical variability in neuroimaging studies. Human Brain Mapping 4, 122-139.

Jiang, A., Kennedy, D.N., Baker, J.R., Weiskoff, R.M., Tootell, R.B.H., Woods, R.P., Benson, R.R., Kwong, K.K., Brady, T.J., Rosen, B.R., Belliveau, J., 1995. Motion detection and correction in functional MR imaging. Human Brain Mapping 3, 224-235.

Kollokian, V., 1994. Performance Analysis of Automatic Techniques for Tissue Classification in Magnetic Resonance Images of the Human Brain. Master's thesis, Dept. of Computer Science, Concordia University, Montreal, Canada.

MacDonald, D., Kabani, N., Avis, D., Evans, A.C., 2000. Automated 3-D extraction of inner and outer surfaces of cerebral cortex from MRI. NeuroImage 12, 340-356. 
Mendola, J.D., Dale, A.M., Fischl, B., Liu, A.K., Tootell, R.B.H., 1999. The representation of illusory and real contours in human cortical visual areas revealed by functional magnetic resonance imaging. J. Neurosci. 19, 8560-8572.

Press, W.A., Brewer, A.A., Dougherty, R.F., Wade, A.R., Wandell, B.A., 2001. Visual areas and spatial summation in human visual cortex. Vision Res. 41, 1321-1332.

Rademacher, J., Caviness Jr., V.S., Steinmetz, H., Galaburda, A.M., 1993. Topographical variations of the human primary cortices: implications for neuroimaging, brain mapping, and neurobiology. Cerebral Cortex 3 , 313-329.

Roland, P.E., Geyer, S., Amunts, K., Schormann, T., Schleicher, A., Malikovic, A., Zille, K., 1997. Cytoarchitectural maps of the human brain in standard anatomical space. Human Brain Mapping 5, 222-227.

Rosa, M.G.P., Soares, J.G., Fiorani Jr., M., Gattass, R., 1993. Cortical afferents of visual area MT in the cebus monkey: possible homologies between new and old world monkeys. Visual Neurosci. 10, 827-855.

Sereno, M.I., Dale, A.M., Reppas, J.B., Kwong, K.K., Belliveau, J.W., Brady, T.J., Rosen, B.R., Tootell, R.B.H., 1995. Borders of multiple visual areas in humans revealed by functional magnetic resonance imaging. Science 268, 889-893.

Sereno, M.I., McDonald, C.T., Allman, J.M., 1994. Analysis of retinotopic maps in extrastriate cortex. Cerebral Cortex 4, 601-620.

Sereno, M.I., Pitzalis, S., Martinez, A., 2001. Mapping of contralateral space in retinotopic coordinates by a parietal cortical area in humans. Science 294, 1350-1354.

Shipp, S., Watson, J.D.G., Frackowiak, R.S.J., Zeki, S., 1995. Retinotopic maps in human prestriate cortex: the demarcation of areas V2 and V3. NeuroImage 2, 125-132.

Sled, J.G., Zijdenbos, A.P., Evans, A.C., 1998. A non-parametric method for automatic correction of intensity non-uniformity in MRI data. IEEE Trans. Med. Imaging 17, 87-97.

Smith, A.T., Greenlee, M.W., Singh, K.D., Kraemer, F.M., Hennig, J., 1998. The processing of first- and second-order motion in human visual cortex assessed by functional magnetic resonance imaging (fMRI). J. Neurosci. 18, 3816-3830.

Steinmetz, H., Fürst, G., Freund, H.J., 1989. Cerebral cortical localization: application and validation of the proportional grid system in MR imaging. J. Comput. Assist. Tomogr. 13, 10-19.

Steinmetz, H., Fürst, G., Freund, H.J., 1990. Variation of perisylvian and calcarine anatomic landmarks within stereotaxic proportional coordinates. Am. J. Neuroradiol. 11, 1123-1130.

Steinmetz, H., Seitz, R.J., 1991. Functional anatomy of language processing: neuroimaging and the problem of individual variability. Neuropsychologia 29, 1149-1161.

Stensaas, S.S., Eddington, D.K., Dobelle, W.H., 1974. The topography and variability of the primairy visual cortex in man. J. Neurosurg. 40 747-755.

Talairach, J., Tournoux, P., 1988. Co-planar Stereotaxic Atlas of the Human Brain. Thieme, New York.
Tootell, R.B.H., Hadjikhani, N., 1998. Has a new color area been discovered? Nature Neurosci. 1, 335-336.

Tootell, R.B.H., Hadjikhani, N., 2001. Where is 'dorsal V4' in human visual cortex? Retinotopic, topographic and functional evidence. Cerebral Cortex 11, 298-311.

Tootell, R.B.H., Hadjikhani, N., Hall, E.K., Marrett, S., Vanduffel, W., Vaughan, J.T., Dale, A.M., 1998a. The retinotopy of visual spatial attention. Neuron 21, 1409-1422.

Tootell, R.B.H., Hadjikhani, N.K., Vanduffel, W., Liu, A.K., Mendola, J.D., Sereno, M.I., Dale, A.M., 1998b. Functional analysis of primary visual cortex (V1) in humans. Proc. Natl. Acad. Sci. USA 95, 811-817.

Tootell, R.B.H., Mendola, J.D., Hadjikhani, N.K., Liu, A.K., Dale, A.M., 1998c. The representation of the ipsilateral visual field in human cerebral cortex. Proc. Natl. Acad. Sci. USA 95, 818-824.

Tootell, R.B.H., Mendola, J.D., Hadjikhani, N.K., Ledden, P.J., Liu, A.K., Reppas, J.B., Sereno, M.I., Dale, A.M., 1997. Functional analysis of V3A and related areas in human visual cortex. J. Neurosci. 17, 70607078 .

Van Essen, D.C., Drury, H.A., 1997. Structural and functional analyses of human cerebral cortex using a surface-based atlas. J. Neurosci. 17, 7079-7102.

Van Essen, D.C., Drury, H.A., Joshi, S., Miller, M.I., 1998. Functional and structural mapping of human cerebral cortex: solutions are in the surfaces. Proc. Nat. Acad. Sci. USA 95, 788-795.

Van Essen, D.C., Drury, H.A., Joshi, S., Miller, M.I., 2000. Functional and structural mapping of human cerebral cortex: solutions are in the surfaces. Adv. Neurol. 84, 23-34.

Voyvodic, J.T., 1999. Real-time fMRI paradigm control, physiology, and behavior combined with near real-time statistical analysis. NeuroImage $10,91-106$

Watson, J.D.G., Myers, R., Frackowiak, R.S.J., Hajnal, J.V., Woods, R.P., Mazziota, J.C., Shipp, S., Zeki, S., 1993. Area V5 of the human brain: evidence from a combined study using positron emission tomography and magnetic resonance imaging. Cerebral Cortex 3, 79-94.

Wong, A.M.F., Sharpe, J.A., 1999. Representation of the visual field in the human occipital cortex-a magnetic resonance imaging and perimetric correlation. Arch. Ophthalmol. 117, 208-217.

Woods, R.P., Cherry, S.R., Mazziotta, J.C., 1992. Rapid automated algorithm for aligning and reslicing PET images. J. Comput. Assist. Tomogr. 16, 620-633.

Woods, R.P., Grafton, S.T., Holmes, C.J., Cherry, S.R., Mazziotta, J.C., 1998. Automated image registration: I. General methods and intrasubject, intramodality validation. J. Comput. Assist. Tomogr. 22, 139-152.

Zeki, S., McKeefry, D.J., Bartels, A., Frackowiak, R.S.J., 1998. Has a new color area been discovered? Nature Neurosci. 1, 335.

Zijdenbos, A.P., Forghani, R., Evans, A.C., 1998. Automatic quantification of MS lesions in 3D MRI brain data sets: Validation of INSECT, in: Proceedings of the First International Conference on Medical Image Computing and Computer-Assisted Intervention (MICCAI), Cambridge, MA, pp. 439-448. 